\title{
Politische Korruption und politischer Wettbewerb: Probleme der quantitativen Analyse*
}

\author{
Philip Manow
}

\section{Einleitung}

Die Beschäftigung mit politischer Korruption gehört zum Kerngeschäft der Politikwissenschaft, weil es hierbei um die zentrale Frage geht, wie das "buon governo" gesichert werden kann. Westliche Demokratien halten auf diese Frage eigentlich eine plausible Antwort bereit. Wer nicht gut regiert, wird abgewählt („throw the rascals out“). Doch offensichtlich funktioniert die demokratische Kontrolle der Regierenden durch die Regierten unterschiedlich gut, wenn man etwa den Corruption Perception Index als ein Performanzkriterium nimmt. Denn selbst wenn wir nur die reifen und reichen Demokratien des Westens in den Blick nehmen, sind wir hinsichtlich der Ausprägung politischer Korruption mit einer großen Variation zwischen den nationalen politischen Systemen konfrontiert, die erklärungsbedürftig ist. Der jährlich ausgewiesene Corruption Perception Index reicht von 0 (vollständig korrupt) bis 10 (vollständig korruptionsfrei). Bereits innerhalb Westeuropas reichte seine Schwankungsbreite im Jahr 2003 zwischen Finnland $(9,7)$ und Griechenland (4,3), erstreckte sich also bereits über mehr als die Hälfte des gesamten Index. Dieser Befund sehr weiter Variation allein schon zwischen den europäischen Demokratien wird in der mittlerweile stark angewachsenen Länderliteratur über politische Korruption bestätigt, ${ }^{1}$ und würde sich noch verstärkt ergeben, wenn wir auch die neuen EU-Mitgliedsländer in die Betrachtung einschließen würden (bspw. mit Polen und Tschechischer Republik mit CPI-Scores von 3,6 bzw. 3,9, um hier ganz zu schweigen von dem Kandidatenland Rumänien). Doch auch schon bei einer Beschränkung auf die etablierten westlichen Demokratien führt der Befund weiter Streuung zur Frage, warum die Kontrollfunktion demokratischer Wahlen anscheinend von Land zu Land so unterschiedlich ausgeprägt ist.

In der Literatur findet man eine große Zahl von Variablen, mit denen politische Korruption zu erklären gesucht wird (Sandholtz and Koetzle 2000; Treisman 2000; Abed and Gupta 2002; Manow 2003; Gerring and Thacker 2004). Unterschiede in nationalen Korruptionslevels werden erklärt 1) mit Unterschieden im ökonomischen Entwicklungsstand, 2) mit Unterschieden im Ausmaß außenwirtschaftlicher Offenheit,

* Ich danke den Teilnehmern der Konferenz „Dimensionen politischer Korruption“ an der Universität Düsseldorf für hilfreiche Kritik und Kommentare. In Teilen rekurrieren die folgenden Ausführungen auf Manow (2003).

1 Diese politikwissenschaftliche Länderliteratur selber ist allerdings nicht Gegenstand der folgenden Darstellung, weil das den Rahmen des Aufsatzes sprengen würde. Ich konzentriere mich im Folgenden auf die theoretisch substanziellen Beiträge sowie auf die quantitativ vergleichenden Studien zum Thema politische Korruption (siehe Treisman 2000; Sandholtz/Koetzle 2000; Sandholtz/Gray 2003; Montinola/Jackman 2002; Persson et al. 2003; Gerring/Thacker 2004; Manow 2003; Abed 2002; Lambsdorff 1999). 
3) in Hinblick auf das Vorkommen von (leicht ausbeutbaren) Rohstoffen, 4) als historische Erbschaft verschiedener Kolonialregime. Korruption wird des Weiteren zurückgeführt auf 5) Unterschiede der Rechtstradition und Rechtskultur (Common Law versus römisches Recht), auf 6) Unterschiede im Niveau der Entlohnung im öffentlichen Sektor, auf 7) Unterschiede im Grad der Freiheit und Unabhängigkeit der Presse, sie wird erklärt mit Unterschieden hinsichtlich 8) dem nationalen Ausmaß sozialer Ungleichheit oder 9) hinsichtlich der zivilgesellschaftlichen Strukturen (soziales Kapital, politische Freiheitsrechte, konfessionelle Zusammensetzung der Bevölkerung), 10) dem Alter der Demokratie etc. pp. Diese Liste ließe sich ohne große Mühe beträchtlich verlängern. Wenn wir uns auf die im engeren Sinne politikwissenschaftlichen Erklärungen konzentrieren, so wird von Politikwissenschaftlern vorzugsweise danach gefragt, ob präsidentielle Systeme korrupter sind als parlamentarische Systeme (Kunicova 2000), ob föderale Länder sich als korruptionsanfälliger erwiesen haben als unitarisch-zentralisierte Länder (Treisman 2000; Fisman/Gatti 2002; Treisman 2002; Gerring/Thacker 2004), ob die Listenwahl eher Korruption befördert als die Persönlichkeitswahl (Golden/Chang 2001; Persson/Tabellini et al. 2001, 2003; Golden 2003), ob konsoziative Demokratien korrupter sind als Konkurrenzdemokratien (Lijphart 1999).

Zentrales theoretisches Konzept in den meisten dieser im engeren Sinne politikwissenschaftlichen Untersuchungen ist der "politische Wettbewerb“. So wird etwa behauptet, dass die Listenwahl c. p. deswegen mit mehr Korruption verbunden ist, weil sie dem Wähler weniger Auswahlmöglichkeiten und daher weniger Sanktionsmöglichkeiten gegenüber dem einzelnen Abgeordneten biete als die Persönlichkeitswahl (Persson, Tabellini et al. 2001, 2003). Gleichfalls begründet sich die Hypothese, dass föderale Systeme weniger korruptionsanfällig seien als unitarisch-zentralisierte Systeme mit dem Argument, dass dezentrale Entscheidungsstrukturen eine unmittelbarere Kontrolle durch die Wähler ermöglichen würden. Im Wettbewerb der Parteien und Kandidaten hätten dadurch "ehrlichere" Wettbewerber elektorale Vorteile. Der gleiche Zusammenhang wird für den Vergleich von Konsensdemokratien und Konkurrenzdemokratien geltend gemacht. Konsoziative Demokratien haben den politischen Wettbewerb zugunsten eines Elitenkartells (Lijphart) oder eines Friedensschlusses zwischen den Lagern und Säulen partiell stillgestellt. Dies, so die These, sollte im Vergleich zu konkurrenzdemokratischen Systemen insgesamt zu mehr politischer Korruption führen.

Viele Debattenbeiträge übernehmen also als ihren theoretischen Ausgangspunkt die ideale Selbstbeschreibung moderner Demokratien, nach der die demokratische Abstimmung ein geeignetes Kontroll- und Sanktionsinstrument ist, wann immer politische Repräsentanten in der Versuchung stehen, ihr Verhalten nicht am Gemeinwohl, sondern am partikularen Eigenwohl auszurichten. Im Rahmen dieses Arguments wird politische Korruption (als „misuse of public office for private gain") mit Defiziten des politischen Wettbewerbs bzw. mit Unterschieden in seiner Effizienz zu erklären gesucht. Miriam Golden und Eric Chang haben diesen Zusammenhang vor kurzem wie folgt prägnant formuliert:

„Schumpeterian competition between political elites ought to prevent large-scale, persistent political corruption from taking root in democratic polities (...) because partisan competitors can always 
offer their service to voters and ought to be preferred over their dishonest counterparts" (Golden/ Chang 2001). ${ }^{2}$

So weit verbreitet und akzeptiert wie dieses Argument ist, so selten sind doch bislang Versuche, es systematisch empirisch auf seine explanative Kraft hin zu untersuchen (Panizza 2001; Montinola/Jackman 2002; Persson, Tabellini et al. 2003).

Dieser Aufsatz möchte den aktuellen Stand der Debatte über die Effekte des politischen Wettbewerbs auf Korruption - kritisch - darstellen. Meine Kritik ist dabei sowohl methodisch als auch theoretisch begründet. Zu diesem Zweck werden ich zunächst die zentralen Thesen der Studien von Persson et al. und Montinola/Jackman kurz darstellen und mit einer Replikationsstudie die Stabilität ihrer Befunde überprüfen (Abschnitt 2). Dabei zeigen sich in meinen Augen Mängel dieser beiden Studien, die Zweifel an ihrem zentralen Ergebnis, dass ein inverser Zusammenhang zwischen politischem Wettbewerb und politischer Korruption besteht, begründen. Diese Zweifel - wohlgemerkt - beziehen sich auf die spezifischen empirischen Befunde und deren theoretische Begründungen. Damit soll nicht zugleich grundsätzlich bestritten werden, dass möglicherweise solch ein inverser Zusammenhang zwischen demokratischem Wettbewerb und politischer Korruption tatsächlich besteht. In einem zweiten Schritt schlage ich vor, die Frage nach dem Verhältnis von politischem Wettbewerb und politischer Korruption in Hinblick auf die Bedeutung von Wahlregeln als effizienten demokratischen Kontroll- und Sanktionsinstrumenten zu stellen. Zu diesem Zweck werde ich den Einfluss von Wahlregeln an einem neuen Datensatz über die Anreize zur Kultivierung eines „personal votes“ überprüfen (Wallack et al. 2002; vgl. Carey/Shugart 1995; Cain et al. 1987). Dabei frage ich, inwiefern das Delegations- und Kontrollverhältnis zwischen Wählern und Parteien effizienter das demokratische Gemeinwohl sichert als das Kontroll- und Delegationsverhältnis zwischen Wählern und Kandidaten (Abschnitt 3). Ich diskutiere zum Schluss einige Folgerungen der Befunde.

\section{Was erklärt politische Korruption? Eine multivariate Betrachtung}

Ich werde im Folgenden diejenigen empirischen Untersuchungen einer näheren kritischen Betrachtung unterwerfen, die meinen, einen negativen Zusammenhang zwischen politischem Wettbewerb und politischer Korruption empirisch bestätigt zu haben (Montinola/Jackman 2002; Persson, Tabellini et al. 2001, 2003). Beide Studien sind relativ neuen Datums und zeigen, dass die Literatur sich erst seit kurzem dem Versuch der systematischen Überprüfung der gängigen Erklärungen für politische Korruption widmet.

Die Studien von Persson et al. sowie Montinola/Jackman streben eine Überprüfung der Hypothese an, dass ein funktionierender politischer Wettbewerb als Remedur gegenüber allen Erscheinungsformen von „improper political practices“ wirkt. Beide Stu-

2 Vom unterstellten Kausalmechanismus anders, aber von der Prognose gleich sind diejenigen Theorien, die der demokratischen Wahl als Funktion nicht das retrospektive Sanktionieren von bad types, sondern das prospektive Selektieren von good types zuschreiben (Fearon 1999). 
dien behaupten, empirische Evidenz für die Geltung dieser Hypothese präsentieren zu können. Persson/Tabellini/Trebbi (künftig PTT) testen die folgenden Hypothesen:

- je größer die Wahldistrikte, desto geringer die Korruption (barriers to entry-Argument): PTT behaupten, dass in größeren Wahlbezirken der Eintritt neuer Kandidaten oder Parteien einfacher, damit der politische Wettbewerb intensiver ist;

- je größer der Anteil der Listenwahl, desto verbreiteter die Korruption (accountability-Argument): PTT behaupten, dass die durch die Parteien erstellten Listen die Auswahlmöglichkeiten für Wähler beschränken, damit den Wettbewerb beschränken, und damit Korruption erleichtern;

- je höher die politische Instabilität, desto höher die Korruption (Kontroll-Argument): PTT postulieren, dass hohe politische Instabilität Korruption fördert, weil sie den Zusammenhang zwischen „politischer Performanz" und retrospektiver Belohnung durch Wiederwahl zerstört; ${ }^{3}$

- Mehrheitswahlrecht in Einzelwahlkreisen sollte weniger Korruption „produzieren“ als das Verhältniswahlrecht in Mehrkandidaten-Wahlbezirken (Wettbewerbs-Argument; vgl. Persson et al. 2001: 5): Erneut steht hinter dieser Hypothese ein Argument höherer und mit schärferen Sanktionsmitteln bewehrter Kontrolle und „accountability“ in Einzelwahlkreisen durch das Mehrheitswahlrecht.

Ich werde im nächsten Abschnitt die theoretische Plausibilität dieser Annahmen detaillierter diskutieren. Hier möchte ich zunächst meine methodische Kritik an der Studie formulieren. Das der Studie von Persson et al. zugrunde liegende Sample umfasst 86 Länder. Über das gesamte Sample finden PTT Bestätigung für ihre Thesen. Die Spalten 1 und 2 in Tabelle 1 berichten ihre Ergebnisse. Dabei wird in Klammern die t-Statistik ausgewiesen. ${ }^{4}$ Wie aus Tabelle 1 ersichtlich, besitzen die Variablen von theoretischem Interesse (Distriktgröße [-], Listenwahl $[+]$ und Instabilität $[+]$ ) die erwarteten Vorzeichen und sind auch statistisch signifikant bzw. nahezu signifikant. (Für die Interpretation muss hervorgehoben werden, dass Persson et al. zur besseren Interpretation den CPI invertiert haben, sodass nun hohe Werte hohe Korruption anzeigen. Negative Koeffizienten bedeuten also in Tabelle 1, dass die betreffende Variable Korruption verringert.)

Bei näherer Betrachtung erweisen sich diese Resultate von PTT jedoch als problematisch. Unter den 86 Ländern des Samples befinden sich 25 Länder, die nach dem Freedom-House-Index lediglich als "teilweise frei“ eingestuft sind, sowie 10 Länder, die nach dem Freedom-House-Index als „unfrei“ gelten müssen. Mit anderen Worten: 41 Prozent des Samples von PTT bestehen aus Ländern, in denen freie Wahlen und basale demokratische Freiheiten nur teilweise gegeben oder sogar vollständig abwesend sind.

3 "Extraction of rents is increasing in political instability, as more instability makes the perceived probability of winning less sensitive to rent extraction" (Persson et al. 2001: 5). Hier ist natürlich auch die genau entgegengesetzte Hypothese plausibel (siehe die Hypothesen $\mathrm{H} 7$ und $\mathrm{H} 7$ ' bei Treisman 2000: 406 und 407): Stabilität verhindert den politischen Wechsel und Wettbewerb und fördert den Aufbau persönlicher Korruptionsnetzwerke. Es scheint, dass Stabilität/ Instabilität am besten als quadratische Funktion integriert werden sollte (siehe hierzu die Überlegungen bei Montinola/Jackman, s.u.). In der 2003er Version haben Persson et al. auf die Betrachtung der Instabilitäts-Variable verzichtet.

4 Als Daumenregel: ein absoluter Wert über 2 zeigt ein Signifikanzniveau von 0,05 oder besser an. 
Tabelle 1: WLS ${ }^{5}$, Abhängige Variable: Corruption Perceptions Index $\varnothing 1997-1999$, Replikation der Persson et al. 2001-Studie (in Klammern t-Statistik)

\begin{tabular}{|c|c|c|c|c|c|}
\hline & 1 & 2 & 3 & 4 & 5 \\
\hline Konstante & $\begin{array}{c}16,23 \\
(10,45)\end{array}$ & $\begin{array}{l}14,33 \\
(7,74)\end{array}$ & $\begin{array}{l}19,04 \\
(4,7)\end{array}$ & $\begin{array}{l}15,93 \\
(7,90)\end{array}$ & $\begin{array}{l}15,61 \\
(2,72)\end{array}$ \\
\hline $\operatorname{LogGDP}$ & $\begin{array}{l}-0,97 \\
(-4,77)\end{array}$ & $\begin{array}{l}-0,85 \\
(-3,95)\end{array}$ & $\begin{array}{l}-1,34 \\
(-3,03)\end{array}$ & $\begin{array}{l}-1,0 \\
(-4,35)\end{array}$ & $\begin{array}{l}-0,28 \\
(-0,41)\end{array}$ \\
\hline LogPop & $\begin{array}{c}0,12 \\
(1,39)\end{array}$ & $\begin{array}{c}0,10 \\
(1,10)\end{array}$ & $\begin{array}{l}-0,03 \\
(-0,21)\end{array}$ & $\begin{array}{c}0,08 \\
(0,76)\end{array}$ & $\begin{array}{l}-0,06 \\
(-0,34)\end{array}$ \\
\hline Education & $\begin{array}{l}-0,02 \\
(-2,18)\end{array}$ & $\begin{array}{l}-0,02 \\
(-2,12)\end{array}$ & $\begin{array}{l}-0,02 \\
(-1,27)\end{array}$ & $\begin{array}{c}-0,02 \\
(-1,90)\end{array}$ & $\begin{array}{l}-0,10 \\
(-2,45)\end{array}$ \\
\hline OECD & $\begin{array}{l}-1,59 \\
(-4,39)\end{array}$ & $\begin{array}{l}-1,58 \\
(-4,59)\end{array}$ & $\begin{array}{l}-0,88 \\
(-1,29)\end{array}$ & $\begin{array}{l}-1,55 \\
(-4,0)\end{array}$ & $\begin{array}{c}0,07 \\
(0,06)\end{array}$ \\
\hline Openness & $\begin{array}{l}-0,01 \\
(-2,73)\end{array}$ & $\begin{array}{l}-0,01 \\
(-2,79)\end{array}$ & $\begin{array}{l}-0,01 \\
(-1,37)\end{array}$ & $\begin{array}{l}-0,01 \\
(-2,7)\end{array}$ & $\begin{array}{c}0,00 \\
(0,02)\end{array}$ \\
\hline $\begin{array}{l}\text { ELF (Ethnisch-linguistische } \\
\text { Fraktionalisierung) }\end{array}$ & $\begin{array}{l}-0,79 \\
(-1,68)\end{array}$ & $\begin{array}{l}-0,80 \\
(-1,75)\end{array}$ & $\begin{array}{l}-0,21 \\
(-0,28)\end{array}$ & $\begin{array}{l}-0,91 \\
(-1,76)\end{array}$ & $\begin{array}{l}-2,28 \\
(-1,71)\end{array}$ \\
\hline Prozent der Protestanten & $\begin{array}{c}-0,02 \\
(3,00)\end{array}$ & $\begin{array}{l}-0,02 \\
(-3,81)\end{array}$ & $\begin{array}{l}-0,02 \\
(-2,08)\end{array}$ & $\begin{array}{l}-0,02 \\
(-3,52)\end{array}$ & $\begin{array}{l}-0,02 \\
(-1,92)\end{array}$ \\
\hline Prozent der Katholiken & $\begin{array}{c}0,01 \\
(2,36)\end{array}$ & $\begin{array}{c}0,01 \\
(1,45)\end{array}$ & $\begin{array}{c}0,01 \\
(2,08)\end{array}$ & $\begin{array}{c}0,01 \\
(1,86)\end{array}$ & $\begin{array}{c}0,01 \\
(0,62)\end{array}$ \\
\hline Prozent der Konfuzianer & $\begin{array}{l}0,3 \\
(0,60)\end{array}$ & $\begin{array}{c}0,50 \\
(0,96)\end{array}$ & $\begin{array}{c}1,78 \\
(1,81)\end{array}$ & $\begin{array}{c}1,02 \\
(1,61)\end{array}$ & $\begin{array}{c}0,72 \\
(0,46)\end{array}$ \\
\hline Listenwahl & & $\begin{array}{c}1,49 \\
(2,67)\end{array}$ & $\begin{array}{c}0,92 \\
(1,27)\end{array}$ & $\begin{array}{c}1,11 \\
(1,87)\end{array}$ & $\begin{array}{c}0,40 \\
(0,3)\end{array}$ \\
\hline Distrikt-Größe & & $\begin{array}{l}-1,10 \\
(-1,67)\end{array}$ & $\begin{array}{c}-0,73 \\
(-0,83)\end{array}$ & $\begin{array}{l}-0,76 \\
(-1,1)\end{array}$ & $\begin{array}{c}-0,8 \\
(-0,49)\end{array}$ \\
\hline Politische Freiheitsrechte & & $\begin{array}{c}0,17 \\
(1,55)\end{array}$ & $\begin{array}{c}0,14 \\
(0,21)\end{array}$ & $\begin{array}{c}0,04 \\
(0,27)\end{array}$ & $\begin{array}{c}1,38 \\
(1,63)\end{array}$ \\
\hline Instabilität & & $\begin{array}{c}0,86 \\
(1,92)\end{array}$ & $\begin{array}{c}0,49 \\
(0,83)\end{array}$ & $\begin{array}{c}0,94 \\
(2,0)\end{array}$ & $\begin{array}{c}-1,13 \\
(-0,89)\end{array}$ \\
\hline $\operatorname{adj} . R^{2}$ & 0,87 & 0,89 & 0,86 & 0,87 & 0,85 \\
\hline Nobs & 81 & 80 & 48 & 68 & 29 \\
\hline Freedom-House Index & & & $\begin{array}{c}<3 \\
\text { (nur freie } \\
\text { Länder) }\end{array}$ & $\begin{array}{c}<5,5 \\
\text { (freie und } \\
\text { teilweise freie } \\
\text { Länder) }\end{array}$ & \\
\hline
\end{tabular}

Reife Demokratie nach Lijphart

Nur reife (1999)

Demokratien

Quelle: Original-Datenset der PTT-Studie, erhältlich auf der persönlichen webpage von Prof. Guido Tabellini unter <http://www.uni-bocconi.it>. Für die Variablenbeschreibung siehe Persson et al. (2001: 27-30).

5 Persson et al. 2001 berichten weighted least squares, wobei als Gewichtung die Inverse der Standardabweichung benutzt wird. Ein solches Vorgehen ist üblich (siehe auch Treisman 2000), um weniger verlässliche Angaben - d.h. Angaben mit hoher Standardabweichung - mit geringerem Anteil in die Schätzung eingehen zu lassen. 
Persson und seine Ko-Autoren haben also den Einfluss des politischen Wettbewerbs auf politische Korruption getestet mit einem Sample, das zu einem erheblichen Teil aus Ländern besteht, in denen von politischem Wettbewerb nur sehr eingeschränkt oder überhaupt nicht die Rede sein kann. Der Freedom-House-Index reicht von 1 (vollständige politische Freiheitsrechte) bis 8 (vollständige Abwesenheit politischer Freiheitsrechte). Folgen wir dem Klassifizierungsvorschlag von Freedom House und bezeichnen Länder mit Scores zwischen 1 und 2,5 als „frei“, Länder mit Scores höher als 3 als "teilweise frei" und Länder mit Scores höher als 5,5 als "unfrei“" (vgl. www.freedomhouse.org/ratings/index.htm), so ergibt eine Replikation der Persson/Tabellini/ Trebbi-Studie für die freien bzw. freien und teilweise freien Ländern die folgenden Ergebnisse (siehe Tabelle 1, Spalten 3 und 4).

Die Vorzeichen der Koeffizienten für die politisch institutionellen Variablen bleiben bei dieser Betrachtung zwar konstant und in der erwarteten Richtung, jedoch verringern sich die Koeffizienten deutlich und verlieren jegliche statistische Signifikanz, sobald wir die Betrachtung auf die vollständig freien Länder einschränken. Keine der Variablen von theoretischem Interesse reicht auch nur an das sehr weiche $\mathrm{p}<10$ Prozent Signifikanzniveau heran. Unter Einschluss auch der „teilweise freien“ Länder verbessern sich die Ergebnisse leicht und die Variablen „Listenwahl" und „politische Instabilität" erreichen nun eine etwas bessere t-Statistik. Dies bleibt jedoch ein problematisches Ergebnis: Die Bestätigung der Hypothese, dass demokratischer Wettbewerb Korruption verringert, fällt umso überzeugender aus, je mehr undemokratische Länder in das Sample integriert werden. ${ }^{6}$ Umgekehrt formuliert: Die Wettbewerbshypothese verfehlt für exakt die Gruppe von Ländern den statistischen Test, für die sie am ehesten Erklärungsanspruch erheben kann - für die Länder mit vollen politischen Partizipationsrechten und einem uneingeschränkt etablierten demokratischen Wettbewerb.

Dieser Befund bestätigt sich, wenn man nur jene Länder in Betracht zieht, die laut Arend Lijphart als etablierte Demokratien gelten können (Lijphart 1999: 48-61). Aus seinem Sample von 36 Ländern, die diesem Kriterium entsprechen, finden sich in dem Ländersample von PTT 29 Länder wieder. Berechnen wir für diese Länder das PTTModell, so ergeben sich die in Spalte 5 berichteten Ergebnisse. Die politisch institutionellen Variablen sind weit von jeglicher statistischer Signifikanz entfernt, für das Sample der i.e.S. demokratischen Länder wechselt sogar die Variable "Instabilität“ ihr Vorzeichen - nun soll angeblich höhere Instabilität Korruption verringern. Insgesamt zeigen die Resultate, dass die Befunde von PTT im Wesentlichen darauf basieren, dass

6 In weiterer Hinsicht sind diese Ergebnisse theoretisch sehr problematisch. Wie vor kurzem erneut von Golden/Chang überzeugend argumentiert wurde, gibt es reichhaltige empirische und theoretische Evidenz für die Annahme, das „personal vote“-Systeme korruptionsanfälliger als Listenwahl-Systeme sind (Golden/Chang 2001, m.w.L.). Die fehlende Unterscheidung bei PTT zwischen offener und geschlossener Listenwahl nährt daher auch erhebliche Zweifel an der Aussagekraft ihrer Befunde (siehe unten). Hinsichtlich der Variable „Instabilität" steht zu vermuten, dass hier erhebliche Endogenitätsprobleme bestehen (d.h. korrupte Systeme sind u.a. deswegen instabiler, weil sie korrupter sind). Schließlich müssen die "Proportionalitä" eines Wahlsystems (Verhältnis von Stimmenanteilen zu Sitzanteilen) bzw. sein "break-even point" (Stimmenanteil, an dem Stimmen und Sitze sich genau entsprechen; vgl. Taagepera/ Shugart 1989) als bessere Indikatoren für die Leichtigkeit des „Markteintritts“ als die Variable „Distriktgröße" angesehen werden. 
die Autoren die „Effekte politischen Wettbewerbs" in Ländern gemessen haben, in denen dieser Wettbewerb nur eingeschränkt oder gar nicht existiert.

Wie sind jedoch nun die Ergebnisse von Montinola und Jackman (2002) zu bewerten, die ebenfalls beanspruchen, empirische Evidenz für die Aussage gefunden zu haben: „political competition reduces corruption“ (S. 151). Montinola und Jackman (künftig MJ) haben das ihren Berechnungen zugrunde liegende Datenset mir freundlicher Weise zur Verfügung gestellt, sodass mir eine exakte Replikation auch ihrer Studie möglich ist. Doch bevor ich die Ergebnisse dieser Berechnungen diskutiere, sollen auch hier vorweg einige kritische Anmerkungen zum Design ihrer Studie formuliert werden. Der erste kritische Einwand bezieht sich auf eine ihrer zentralen unabhängigen Variablen. Es bleibt bei MJ eigentümlich unklar, welche theoretische Hypothese die Autoren eigentlich genau testen wollen. MJ sprechen zwar vom Einfluss der ,competition in the political arena" (S. 147) auf Korruption, testen aber nicht diese Hypothese mit einer der vielfältigen Wettbewerbsvariablen, wie sie in der Literatur vorgeschlagen werden (Strøm 1989, 1990). Stattdessen nehmen sie einen der vielen Indikatoren für politische Freiheitsrechte. Damit wird aber eben nicht der Effekt des tatsächlichen Wettbewerbs gemessen, sondern nur, ob für ihn die institutionellen und rechtlichen Voraussetzungen grundsätzlich bestanden haben. Um ein Beispiel zu nennen: Das politische System Großbritanniens und das von Italien oder Japan können für die gesamte Nachkriegsperiode als vollständig demokratisiert charakterisiert werden. Es ist daher nicht überraschend, dass alle drei Länder auf dem von MJ verwendeten Demokratie-Indikator von Kenneth Bollen mit 100 identisch eingestuft werden (Bollen 1993: 1227). Doch ohne Zweifel unterscheidet sich das Ausmaß effektiven politischen Wettbewerbs ganz erheblich zwischen diesen Ländern - wie immer man politischen Wettbewerb auch misst (vgl. Strøm 1989, 1990). ${ }^{7}$ Mit anderen Worten: Die Studie von Montinola und Jackman sagt überhaupt nichts aus zur eigentlich theoretisch interessanten Frage: Können Unterschiede in der Intensität des demokratischen Wettbewerbs Unterschiede im Ausmaß der Korruption erklären? Die einzige Frage, zu der sie eine Antwort liefern können, lautet: Macht es einen Unterschied für politische Korruption, ob basale demokratische Rechte in einem Land gewährt sind oder nicht?

In der von MJ untersuchten Modellvariante sind die Autoren auch mit einem weiteren theoretischen Problem konfrontiert: Es ist ja nicht auszuschließen, dass bestimmte Länder nur deswegen volle demokratische Rechte gewähren, weil der tatsächliche demokratische Wettbewerb grundlegend defizitär, z.B. systematisch verzerrt ist. Mit anderen Worten: Volle Gewährleistung der formalen politischen Partiziparionsrechte kann möglicherweise in einem Land deswegen vorgefunden werden, weil bspw. sein Wahlund Parteiensystem die systematische Bevorzugung einer spezifischen Oligarchenschicht oder bestimmter politischer Eliten garantiert. Alles spricht also dafür, dass mit den

7 Als Veranschaulichung: Hinsichtlich der bei Strøm berichteten Werte zur Alternierung und Responsivität politischer Systeme belegen Großbritannien und Italien Extrempositionen. Auf der von 0 bis 1 reichenden Alternierungsskala liegt Italien bei 0,04, während Großbritannien einen hohen Wert von 0,37 erreicht. Responsivität, als eine Maßzahl für die Wahrscheinlichkeit, mit der eine Stimmen hinzugewinnende Partei in der nächsten Regierung vertreten sein wird, hat in Großbritannien den Wert 1, in Italien den Wert 0,45. Die Gegenüberstellung ließe sich beliebig fortsetzen. 
gängigen Indizes für politische Freiheitsrechte tatsächlicher politischer Wettbewerb überhaupt nicht gemessen werden kann. Insofern können auch die Autoren nicht beanspruchen, Evidenz für die Aussage "political competition matters" (S. 167) gefunden zu haben.

Das Design der Studie von MJ setzt sich weiterer methodischer Kritik aus. Erstens verwenden die Autoren einen veralteten und wenig verlässlichen Korruptionsindikator, den Business International Indicator (BI-Index). Sie kontrollieren zwar ihre Studie durch die Verwendung des Corruption Perceptions Index, jedoch nehmen sie hier die 1988-1992 Angaben von Transparency International (TI), obwohl TI seitdem mehr als acht Nachfolgeerhebungen vorgenommen hat. Eine Korrelationstabelle zwischen den CPI-Scores verschiedener Jahrgänge zeigt, dass die 1988-1992 Angaben vergleichsweise geringe Verlässlichkeit besitzen (Manow 2002). Das bei MJ wiederholt gegen den Index von Transparency International vorgebrachte Argument der geringen Fallzahl (S. 157 „principal drawback ... is its more restricted country coverage“; siehe ebenso 165) fiele in sich zusammen, hätten die Autoren die neueren Surveys von TI berücksichtigt. Der 2002 CPI umfasst 102 Länder, bereits der 1999er Index - volle zwei Jahre vor der Veröffentlichung der Studie von MJ frei zugänglich - umfasste mit 99 Ländern 33 Länder mehr als der von den Autoren favorisierte BI-Index. Samplegröße ist also kein Argument, das für den ansonsten wenig verlässlichen BI-Index ins Feld geführt werden kann. Da für alle anderen unabhängigen Variablen der Studie auch aktuelle Maßzahlen frei verfügbar sind, sprechen keinerlei Gründe gegen die Verwendung der neuesten TI-Maßzahlen.

Schließlich beanspruchen MJ auch noch, den Einfluss der relativen Einkommensposition öffentlich Bediensteter zu testen. Hier allerdings behaupten sie, „no data are available for either number of public-sector employees or specific expenditures on wages, [so] we cannot directly address this issue" (S. 158). Sie verwenden daher "level of economic development" (natürlicher Logarithmus des realen BIP pro Kopf) als Näherungswert für die Löhne im öffentlichen Sektor (S. 154). Eine nähere Diskussion über diesen ausgesprochen kruden Annäherungswert erübrigt sich, da die Behauptung, es gäbe keine international vergleichbaren Angaben zu den Löhnen im öffentlichen Sektor, nicht zutrifft (siehe die Studie von Schiavo-Campo et al. 1997). Wären die Autoren mit der grundlegenden vergleichenden Studie von Treisman über die "Causes of Corruption" (2000) vertraut gewesen, so wäre ihnen dieser Fehler nicht unterlaufen, denn Treisman berichtet die Daten von Schiavo-Campo. Dass Treisman in seiner multivariaten Untersuchung ebenso wie Schiavo-Campo et al. einen statistisch signifikanten Einfluss von Beamtenlöhnen auf Korruption nicht feststellen konnte, MJ aber einen solchen zu finden glauben (S. 169), wirft somit ein schlechtes Licht auf die Konstruktvalidität ihres Proxy-Wertes „wirtschaftlicher Entwicklungsstand“". Diese Vermutung bestätigt sich, wenn man die Angaben von Schiavo-Campo et al. mit den Angaben zum Pro-Kopf-BIP bei MJ korreliert. Der Korrelationskoeffizient ist mit 0,55 unter dem Niveau, der als Anforderung an eine instrumentelle Variable gestellt werden muss - und negativ! Mit anderen Worten, entgegen der nicht näher begründeten Annahmen bei MJ, dass die relative Entlohnung der Staatsdiener (gemessen als Prozentteil des GDPs oder als Prozentteil des Pro-Kopf-GDPs) je höher ist, desto reicher ein Land ist (S. 169: „substantial correlation [of GDP] with average wages, both public and pri- 


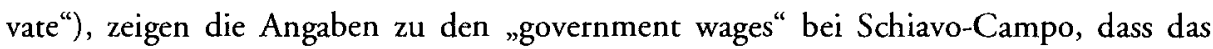
Gegenteil der Fall ist: Ärmere Länder entlohnen ihre Staatsbeschäftigten relativ höher als reiche Nationen. Als Schlussfolgerung ergibt sich daher bereits vor einem Blick auf die Daten, dass nichts an der Analyse von MJ ihre resümierende Behauptung rechtfertigt: "higher wages ... reduce the incentives for corruption“ (S. 169).

Welcher empirische Befund ergibt sich nun unabhängig von dieser Kritik am Design der Studie von Montinola und Jackman, wenn wir ihr Erklärungsmodell unter Einschluss der Daten zu der Lohnhöhe im öffentlichen Sektor replizieren?8 Tabelle 2 berichtet die Ergebnisse meiner Replikation. Die Spalten 1 und 2 geben die originalen Befunde von MJ wieder, Spalte 3 enthält die Ergebnisse der Neuberechnung unter Verwendung der Angaben zur Entlohnung der Staatsbediensteten.

Tabelle 2: Replikation der MJ-Studie (in Klammern t-Statistik)

\begin{tabular}{lcccc}
\hline Abhängige Variable: & & & & \\
Business International Index 1980-83 & 1 & 2 & 3 & 4 \\
\hline Konstante & $-13,32$ & $-11,05$ & 8,72 & $-12,48$ \\
& $(4,9)$ & $(3,83)$ & $(3,97)$ & $(-3,10)$ \\
Demokratie 80 & 0,08 & 0,15 & 0,37 & 0,09 \\
& $(1,683)$ & $(2,49)$ & $(4,61)$ & $(1,22)$ \\
Demokratie ${ }^{2} 80$ & & 0,04 & 0,096 & 0,04 \\
& & $(1,88)$ & $(3,08)$ & $(1,70)$ \\
Staatsquote & 1,57 & 1,31 & $-1,13$ & 1,04 \\
& $(2,96)$ & $(2,46)$ & $(-1,38)$ & $(1,44)$ \\
Pro-Kopf BlB & 1,88 & 1,68 & & 1,88 \\
& $(8,61)$ & $(7,00)$ & & $(5,79)$ \\
Löhne im öffentlichen Sektor $\approx 1990-1995$ & & & 0,08 & 0,21 \\
& & & $(0,43)$ & $(1,43)$ \\
OPEC & $-1,97$ & $-1,78$ & $-1,54$ & $-1,38$ \\
& $(3,93)$ & $(3,54)$ & $(-1,70)$ & \\
\# Nobs & 66 & 66 & 50 & 50 \\
R2 & 0,66 & 0,67 & 0,43 & 0,67 \\
(F-ratio) & $(32,36)$ & $(27,67)$ & $(8,49)$ & $(17,88)$ \\
\hline
\end{tabular}

Quelle: Montinola/Jackman (2002) und Schiavo-Campo et al. (1997).

Die Ergebnisse sind vergleichsweise leicht zu interpretieren. Im Gegensatz zu der von MJ verwendeten Variablen des Pro-Kopf BIPs erweist sich die Lohn-Variable als nicht erklärungskräftig. Die OPEC-Variable verliert ebenfalls ihre Signifikanz. ${ }^{9}$ Auf den ers-

8 Die Daten von Schiavo-Campo et al. spiegeln den Stand der frühen 1990er wider, der BIIndex von Montinola/Jackman den Stand der frühen 1980er Jahre. Da es sich hier aber um Daten handelt, die nicht großen kurzfristigen Schwankungen unterworfen sind, scheint mir eine Integration der Lohndaten in das Modell von MJ als weitaus weniger problematisch als die Verwendung eines so kruden Annäherungswertes wie dem des Pro-Kopf-BIP.

9 Dies ist in erster Linie ein Variableneffekt, und kein durch die Verringerung der Samplegröße (von 66 auf 50) hervorgerufener Effekt. Denn bei Beschränkung auf die 50 Länder aus dem MJ-Sample, für die vergleichbare Zahlen zur Entlohnung der Staatsbediensteten vorliegen, 
ten Blick scheint sich dagegen die Erklärungskraft der Demokratie-Variablen deutlich zu erhöhen (erhöhter Koeffizient, verbesserte t-Statistik). Dass dies jedoch allein ein Effekt des Ausschlusses der stark signifikanten Variable Pro-Kopf BIP ist, zeigt sich in Spalte 5. In den ersten beiden Modellspezifikationen von MJ hatte diese Variable ja sehr große Erklärungskraft bewiesen. Sie hatte das aber nicht als Annäherungswert an den vermuteten Kausalfaktor "Lohnhöhe im Staatsdienst“, wie sich bei Einschluss der Informationen zu der Lohnhöhe zeigt. In bislang allen komparativen Untersuchungen hatte sich ein "Reichtumseffekt" auf das Niveau der politischen Korruption als stabil und erklärungskräftig erwiesen. Genau diesen Reichtums- oder Modernisierungseffekt misst die Pro-Kopf BIP Variable. Trägt man deren Einfluss Rechnung (Spalte 5), so zeigt sich, dass der von MJ behauptete Einfluss von Demokratie auf Korruption weitgehend verschwindet. Um es zusammen zu fassen: MJ messen etwas anderes (demokratische Freiheitsrechte), als sie zu messen vorgeben (politischen Wettbewerb), und selbst das, was sie messen, hat nicht den von ihnen behaupteten Einfluss auf die abhängige Variable - politische Korruption - sowie wir ihre eigene Theorie und den bisherigen Forschungsstand zum Thema ernst nehmen.

Da MJ einen veralteten, wenig verlässlichen Korruptions-Index verwenden, scheint es auch empfehlenswert, ihr Modell mit aktuelleren Daten erneut zu rechnen. Für diese Neuberechnung verwende ich statt des Business International Index den 1998er CPIndex als abhängige Variable. Der Proxy für das Lohnniveau öffentlicher Beschäftigter wird erneut durch die direkten Angaben über die staatlichen Ausgaben für Löhne und Gehälter ersetzt (Schiavo-Campo et al. 1997; gemessen als Prozent des BIP). Statt des von MJ verwendeten Indikators für das Ausmaß der Gewährung politisch-demokratischer Freiheitsrechte verwende ich den Freedom House Index für das Jahr 1998. Darüber hinaus habe ich die Angaben von MJ zur Variable "Staatsquote" (Penn World Table 6.0) für das Jahr $1997 / 98$ aktualisiert. Wie MJ habe ich ebenfalls eine Dummy-Variable für OPEC-Mitgliedschaft integriert, obwohl mit nur 3 OPEC-Mitgliedern diese Variable in dem von mir verwendeten Sample keine große Rolle spielt. Die Fallzahl des aktualisierten Modells liegt mit 61 Ländern dicht an der Samplegröße von MJ $(\mathrm{N}=66),{ }^{10}$ hat nun aber den Vorteil einer weitaus verlässlicheren abhängigen Variablen (1998er CP-Index) und einer nicht nur als grober Annäherungswert integrierten Variable über die relative Entlohnungsposition öffentlicher Bediensteter (siehe Tabelle 3).

Folgende Ergebnisse, die im Widerspruch zu den Befunden von MJ stehen, möchte ich hervorheben: Zunächst erweist sich der Effekt der relativen Lohnhöhe öffentlich Bediensteter als - wenn überhaupt erklärungskräftig - dann negativ. höhere Löhne erhöhen das Korruptionsniveau (vermindern den CPI-Wert). Zu dem gleichen Ergebnis kommen Schiavo-Campo et al. (1997). Treisman berichtet für diese Variable insignifikante, aber positive Koeffizienten (2000). Die betreffende Variable hat in Tabelle 3 durchweg ein negatives Vorzeichen, ist jedoch nicht statistisch signifikant. MJ berichte-

bleiben die Variablen Pro-Kopf-BIP und OPEC-Mitgliedschaft nahezu unverändert (Berechnungen sind hier nicht berichtet).

10 Diese eingeschränkte Samplegröße ergibt sich nicht wegen der Verwendung des CP-Indexes, vielmehr ist das Sample dadurch begrenzt, dass Angaben über die Löhne im öffentlichen Sektor für viele Länder fehlen. Trotzdem übertrifft die hier einbezogene Fallzahl die meisten Modelle in der grundlegenden Studie von Treisman (2000). 
Tabelle 3: Neuberechung des Montinola/Jackman-Modells mit aktuellen Daten (in Klammern t-Statistik)

\begin{tabular}{lcccc}
\hline Abhängige Variable: CPI 1998 & 1 & 2 & 3 & 4 \\
\hline Konstante & 14,01 & 12,66 & 9,87 & 12,54 \\
& $(2,17)$ & $(2,32)$ & $(2,00)$ & $(2,3)$ \\
Demokratie 80 & $-0,79$ & $-1,27$ & $-1,20$ & $-1,22$ \\
& $(-5,18)$ & $(-7,87)$ & $(-6,89)$ & $(-7,00)$ \\
Demokratie ${ }^{2} 1998$ & & 0,379 & 0,354 & 0,356 \\
& & $(4,92)$ & $(4,33)$ & $(4,37)$ \\
Staatsquote 1998 & 11,88 & 13,07 & $-1,40$ & 12,67 \\
& $(0,80)$ & $(1,04)$ & $(-1,17)$ & $(1,01)$ \\
Pro-Kopf BIP & $-14,04$ & $-15,19$ & & $-14,68$ \\
& $(-0,91)$ & $(-1,17)$ & & $(-1,12)$ \\
Löhne im öffentlichen Sektor $\approx 1990-1995$ & & & $-0,1$ & $-0,09$ \\
& & & $(-0,89)$ & $(-0,84)$ \\
OPEC & $-1,65$ & $-1,23$ & $-1,66$ & $-1,38$ \\
& $(-1,36)$ & $(-1,19)$ & $(-1,62)$ & $(-1,32)$ \\
\# Nobs & 61 & 61 & 61 & 61 \\
$\mathrm{R}^{2}$ & 0,37 & 0,55 & 0,55 & 0,55 \\
\hline
\end{tabular}

Quellen: Freedom House Index; Penn World Tables 6.0; Schiavo-Campo et al. (1997).

ten für ihren Annäherungswert Pro-Kopf BIP durchweg ein positives Vorzeichen (siehe Montinola/Jackman 2002: 160, Table 1 und 166, Table 3). Zudem sind die beiden Demokratievariablen in allen neu berechneten Modellspezifikationen sehr viel stärker signifikant als in dem originalen MJ-Modell. Zudem zeigen die Berechnungen mit den aktualisierten Daten, dass die Beziehung zwischen politischen Freiheitsrechten und Korruption nicht-linear ist. Der direkte Zusammenhang zwischen der einfachen Demokratie-Variable und der abhängigen CPI-Variablen ist negativ (und signifikant, während er bei $\mathrm{MJ}$ durchweg positiv, aber insignifikant ist). Die Demokratie ${ }^{2}-$ Variable erweist sich als positiv und dabei ebenfalls als robust erklärungskräftig. Das scheint zunächst eine Bestätigung der Vermutung bei MJ zu sein: „the effect of democracy on corruption is nonlinear" (S. 161). Doch während die Schätzung von MJ einen exponentiellen Zusammenhang vorhersagt, legt meine Neuberechnung ihres Modells einen quadratischen Zusammenhang nahe (siehe Abbildung 1). Dabei passen diese neu berechneten Befunde weit besser zur Interpretation der Autoren als ihre eigenen Daten, denn folgt man MJ, so ist die Korruption in mittel-demokratisierten Ländern höher als in Ländern mit autoritären Regimen (S. 167). Ihre eigenen Resultate hingegen implizieren, dass mehr Demokratie immer zu weniger Korruption führt (sowohl die einfache, als auch die quadrierte Demokratie-Variable haben positive Vorzeichen!; MJ 2002: 160, Table 1, 162 Table 2). Diesen manifesten Widerspruch zwischen ihren Ergebnis und ihrer Interpretation thematisieren die Autoren nicht weiter.

Die äußerst geringe Robustheit aller anderen Befunde von $\mathrm{MJ}$ gegenüber leichten Veränderungen im Ländersample oder gegenüber marginalen Veränderungen in der Operationalisierung der unabhängigen Variablen muss ausgesprochen skeptisch stimmen hinsichtlich der Verlässlichkeit ihrer inhaltlichen Schlussfolgerungen. Unbenom- 
Abbildung 1: Vergleich der Prognose des MJ Schätzers und des neu berechneten Schätzers, Tabelle 2 Spalte 2 und Tabelle 3, Spalte 4

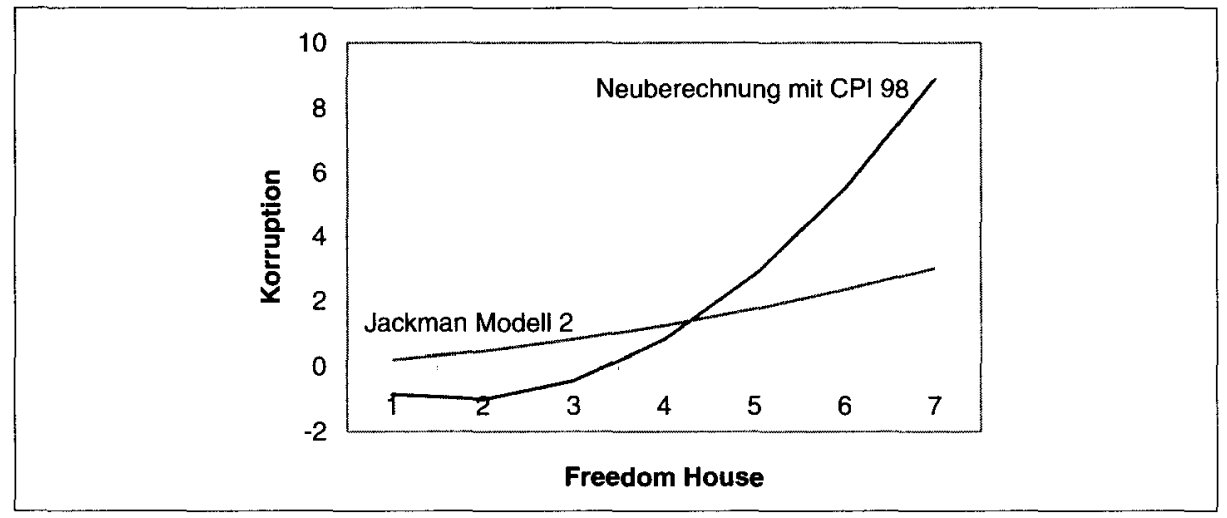

men von dieser generellen Skepsis bleibt die grundlegende Kritik, dass MJ nicht - wie von ihnen selbst behauptet - den Einfluss politischen Wettbewerbs auf Korruption untersucht haben, sondern den Einfluss der Gewährung politischer Freiheits- und Partizipationsrechte auf Korruption. Dies ist allerdings eine gänzlich andere Sache.

\section{Politischer Wettbewerb und Wahlregeln: eine Überprïfung der "personal vote"-These}

Aus der vorangegangenen Argumentation ergibt sich u.a. die folgende Einsicht: Die verschiedenen vergleichend-quantitativen Arbeiten zum Einfluss von Wahlregeln auf Korruption haben bislang lediglich zu Resultaten geführt, die als „highly equivocal“ bezeichnet werden müssen (Gerring/Thacker 2004: 298). Insbesondere umstritten bleibt die Frage, ob die Listenwahl oder die Persönlichkeitswahl ein effizienteres demokratisches Kontrollmittel darstellt (siehe oben, Fn. 6). Eine breite politikwissenschaftliche Literatur verweist darauf, dass der wichtige Unterschied in diesem Zusammenhang nicht darin besteht, ob nach Listen oder nach Personen abgestimmt wird, sondern ob die Rangfolge auf Listen durch den Wähler verändert werden kann (offene Listen) und ob "persönliche Stimmen" auf andere Kandidaten übertragbar sind oder nicht (Carey/ Shugart 1995; Nohlen 2000; Shugart 2001). So ist das hohe Ausmaß der politischen Korruption in Italien plausibel mit dem italienischen Wahlsystem der offenen Listen und Mehrfachstimmen (voto di preferenza) in Verbindung gebracht worden (Golden/ Chang 2001; Golden 2003), das die partei-interne Konkurrenz insbesondere innerhalb der DC angeheizt, aber dadurch zugleich den Wettbewerb zwischen den Parteien stark gemindert hat. Eine ähnliche Wirkung wird dem ganz anders funktionierenden japanischen Wahlsystem mit dem Single Nontransferable Vote in Mehrkandidatenwahlkreisen zugeschrieben. Hier ist es nicht der Kampf um gute Rangplätze auf offenen Listen, sondern der Ausschluss der Übertragbarkeit von Stimmen auf andere Kandidaten der gleichen Partei, der einen starken Anreiz zur Etablierung (teurer) persönlicher Unterstützungsnetzwerke mit ihrer allgegenwärtigen Tendenz zu korrupten Praktiken darstellt. Diesen Feinheiten des Wahlsystems wird man aber durch die simple Gegenüber- 
stellung von Listenwahl und Persönlichkeitswahl nicht gerecht, wie sie etwa in der PTT-Studie vorgenommen worden ist.

Seit kurzem liegt allerdings ein vergleichender Datensatz mit recht hoher Fallzahl vor, der die grundlegenden Überlegungen von Carey und Shugart zu den Anreizeffekten von Wahlregeln (1995) systematisch aufgenommen hat und den Kodierungsvorschlägen dieser Autoren weitgehend gefolgt ist (Wallack et al. 2002). Dieser Datensatz ermöglicht eine sehr viel genauere Überprüfung der "demokratischen Effizienz" verschiedener Wahlregeln, als dies mit den bislang vorgelegten Untersuchungen möglich war, weil er die spezifischen Anreizwirkungen einzelner elektoraler Regeln deutlich verbessert abbildet. Carey und Shugart hatten in ihrer einflussreichen Untersuchung über Anreize zur Ausbildung eines "personal vote“ drei Dimensionen von Wahlregeln unterschieden, die alle bei der Frage eine entscheidenden Rolle spielen, ob ein Wahlsystem eher parteien-orientiert oder eher kandidaten-orientiert ist, ob eher die Parteireputation oder die persönliche Kandidatenreputation für die Wahl ausschlaggebend ist. Sie nannten diese drei Dimensionen Ballot, Pool, Vote. Zunächst - so die Autoren - ist entscheidend, wie stark eine Partei beeinflussen kann, wer sich unter ihrem Label um politische Ämter bewirbt (Ballot). Dann ist entscheidend, wie sehr Stimmen zwischen Kandidaten ein und derselben Partei "gepoolt" werden oder aber ausschließlich für einzelne Kandidaten zählen (Pool). Schliesslich ist von Bedeutung, ob Wähler eine oder mehrer Stimmen haben und ob sie mit diesen Stimmen Präferenzen über Parteien oder über Kandidaten ausdrücken können (Vote; Carey/Shugart 1995: 420-423). Jede dieser drei Dimensionen hat bei Carey und Shugart drei Ausprägungen, die von 0 (partei-kontrollierte Listenerstellung, volles Verrechnen von Stimmen zwischen Kandidaten und Einzelstimme für Parteiliste) bis 2 (freie Kandidatenbewerbungen, kein Verrechnen und Einzelstimmen für Einzelkandidaten) reichen. Diese Überlegungen sind für die Erstellung des Datensatzes „Political Particularism around the World" mit geringfügigen Modifikationen übernommen worden (Wallack et al. 2002). ${ }^{11} \mathrm{Im}$ Folgenden möchte ich nun mit Hilfe dieses Datensatzes insbesondere die umstrittene Frage wieder aufgreifen, inwiefern die Listen- bzw. die Persönlichkeitswahl eine bessere Kontrolle der Regierenden durch die Regierten ermöglicht.

Bevor ich mein methodisches Vorgehen knapp erläutere, sind jedoch zwei inhaltliche Anmerkungen über den mit dem gewählten Vorgehen verbundenen Vorteil (a) bzw. Nachteil (b) voraus zu schicken. (a) Der Frage nach dem Einfluss des politischen Wettbewerbs auf Korruption anhand der Untersuchung von Wahlregeleffekten nachzu-

11 Carey und Shugart behandeln als vierte wichtige Dimension die Größe des Wahlbezirks. Diese hat aber unterschiedliche Wirkungen je nachdem, ob Wähler zwischen Kandidaten auswählen können oder nicht. In Systemen mit geschlossener Liste nimmt die Bedeutung persönlicher Reputation mit zunehmender Größe des Wahlbezirks ab. Bei Persönlichkeitswahl oder offenen Listen nimmt die Bedeutung einer persönlichen Reputation mit zunehmender Größe des Wahlbezirks hingegen zu (1995: 430-432). Es handelt sich hier jedoch um zwei verschiedene Arten von Reputation, und im Kontext meiner Überlegungen spielen nicht beide eine Rolle. Einmal geht es um eine lokale Reputation (a reputation to deliver), das andere Mal geht es um eine überregionale Bekanntheit oder Berühmtheit, die nichts mit den politischen Fähigkeiten zu tun hat, spezifische persönliche Vorteile zu gewähren und partikularen, lokalen Interessen zu dienen. Da hier nur das Erste interessiert, vernachlässige ich im Folgenden die Größe des Wahldistrikts. 
gehen, hat meines Erachtens den Vorteil, dass wir damit vermeiden, Indikatoren zu wählen, die immer schon das Resultat des Zusammenspiels von institutionellen Regeln, Interessenlagen und strategischen, auf den institutionellen Rahmen reagierenden Verhaltens sind. Nimmt man stattdessen einen der in der Literatur gängigen Wettbewerbsindikatoren wie „closeness" (Abstand der Stimmenanteile zwischen der stärksten und zweitstärksten Partei) oder "responsiveness“ (Wahrscheinlichkeit, nach der Parteien mit Stimmenzugewinnen in der Regierung vertreten sein werden; vgl. als Übersicht Strøm (1989, 1990) oder die effektive Zahl der Parteien (Rae 1971) so haben wir immer Ergebnisindikatoren, bei denen die exklusive Zurechnung auf Institutionen, Interessen oder Strategien unmöglich ist. (b) Der Nachteil des gewählten Vorgehens ist jedoch, dass wir natürlich nur einen isolierten Einzelaspekt betrachten, und damit gerade „institutionelle Interaktionseffekte" von hohem theoretischen Interesse nicht in den Blick bekommen. Um ein Beispiel für unseren Kontext zu geben: Wie Gary Cox überzeugend argumentiert hat, ist das sehr hohe Ausmaß an Exekutivdominanz im britischen Westminster-System historisch gerade als Antwort auf die hohen Anreize zum partikularistischen Abgeordnetenverhalten zu verstehen, die im britischen Mehrheitswahlrecht in Einzelwahlkreisen begründet sind (Cox 1987). Wenn wir also allein den Effekt von Wahlregeln untersuchen, entgeht uns womöglich, dass wir in den untersuchten politischen Systemen bereits schon institutionell verfestigte Antworten auf die Folgeprobleme bestimmter Wahlregeln vorfinden. Für die nachstehende empirische Untersuchung gilt deswegen die gleiche einschränkende Bemerkung, die auch Carey und Shugart selbst im Kontext ihrer Untersuchung betonen: ihr "rank-ordering“ der Anreize zur Ausbildung einer persönlichen Kandidatenreputation "pertains strictly to electoral systems, rather than to government systems as a whole" (Carey/Shugart 1995). Von vornherein ist damit die Erwartung gedämpft, empirisch starke Zusammenhänge zwischen Wahlregeln und demokratischen Performanzkriterien wie etwa Korruptionswerten zu finden, weil dieser Zusammenhang immer auch noch durch den Einfluss anderer Regierungsinstitutionen gebrochen ist. Was im Lichte dieser Überlegungen als wünschenswert erscheint, wäre die Integration von institutionellen Interaktionseffekten, aber der Stand der Debatte im Bereich der "comparative political institutions" ist noch nicht hinreichend entwickelt, um über die Untersuchung des Einflusses einzelner politischer Institutionen - hier Wahlregeln - hinaus zu gehen.

Was das Design meiner folgenden kurzen empirischen Untersuchung angeht, so folge ich bei meinen Berechnungen im Wesentlichen den Befunden bei Treisman (2000), Sandholtz/Koetzle (2000), Sandholtz/Gray (2003), Persson/Tabellini/Trebbi (2003) und Gerring/Thacker (2004) und kontrolliere im Regressionsmodell für Bruttosozialprodukt per capita, britisches Kolonialerbe und protestantischen Bevölkerungsanteil. In ganz verschiedenen Modellspezifikationen haben sich diese drei Variablen immer wieder als robust erklärungskräftig erwiesen. So auch bei meinen Berechnungen. Ich schließe nur Demokratien ein, die im Freedom House Index einen Wert von 3 oder besser zugewiesen bekommen haben. Insgesamt halte ich es für theoretisch überzeugender, den Einfluss des demokratischen Wettbewerbs auch wirklich an der Gruppe von Ländern zu überprüfen, die volle demokratische Freiheitsrechte gewähren. Selbst bei dieser restriktiven Bedingung liegt die Fallzahl immer noch bei 47 Ländern, was das Testen einfacher Modelle mit einer beschränkten Zahl von erklärenden Variablen 
erlaubt. Schließlich berechne ich - wie die meisten der oben genannten Studien (vgl. Fn. 5) - ebenfalls Weighted Least Squares, d.h. gewichte die abhängige Variable mit ihrer Standardabweichung, um Werten mit größerer Varianz in der Regression eine geringeres Gewicht zuzuweisen.

Die abhängige Variable der folgenden Regressionen ist der Corruption Perception Index von Transparency International aus dem Jahr 2001. Da der Index sowohl mit den Indexen der Vorjahre als auch mit den neueren TI-Indexen eine sehr hohe Korrelation aufweist, hat die Wahl des Jahres selbst keine nachhaltigen Auswirkungen auf die Ergebnisse. Berechnungen mit dem TI-Index aus anderen Jahren (oder auch mit anderen Korruptions-Indizes; vgl. Kaufmann et al. 2002) brachten im Wesentlichen dieselben Resultate. Neben den genannten Kontrollvariablen habe ich als Variablen von theoretischem Interesse sowohl die Ausprägungen von Ballot, Pool und Vote als auch einen aus der Addition der drei Variablen sich ergebenden Indexwert (Minimum 0 , Maximum 6) untersucht. Der Datensatz „Political Particularism around the World“ deckt den Zeitraum von 1978 bis 2001 ab. Die Regressionsberechnungen erfolgten sowohl mit den aktuellen Werten, als auch mit den Durchschnittswerten der Jahre 1978-1990. Hinter der Verwendung älterer Durchschnittszahlen steht die Überlegung, dass die Effekte von Wahlregeln möglicherweise vor allem langfristiger Natur sind eine Änderung im Wahlsystem führt nicht zu unmittelbaren Änderungen im Verhalten der politischen Akteure, weil die Strategien und Strukturen von korporativen Akteuren wie politischen Parteien meistens nicht von heute auf morgen zu ändern sind. Da die drei Variablen sowohl untereinander als auch mit dem addierten Index eine hohe Kollinearität aufweisen (Ballot/Pool 0,6; Ballot/Vote 0,74; Pool/Vote 0,63; vgl. Wallack et al. 2002: 4), habe ich sie jeweils einzeln in die Regression integriert. Die Ergebnisse sind in Tabelle 4 berichtet, wobei hier nur die Ergebnisse der Berechnungen mit den aktuellen Werten aus dem Partikularismus-Datensatz wiedergegeben werden.

Was zunächst an Tabelle 4 auffällt ist, dass die Koeffizienten von Ballot, Pool und Vote sowie auch der Koeffizient des addierten Index durchgängig negative Vorzeichen aufweisen. Sie sind leicht bis deutlich signifikant. In die gleiche Richtung - bei Verschiebungen der Gewichte zwischen den einzelnen Komponenten - weisen die Ergebnisse, wenn man die 1978-1990 Durchschnittswerte für die erklärenden Variablen Ballot, Pool und Vote bzw. der aggregierten Variablen nimmt. Auch hier weisen die Koeffizienten von theoretischem Interesse über alle Modellspezifikationen hinweg durchweg negative Vorzeichen auf. Mit anderen Worten: Die Regressionsbefunde - so vorsichtig, wie sie zu interpretieren sind - stützen doch durchgängig die Hypothesen der politikwissenschaftlichen „personal vote"-Literatur (Cain et al. 1987): je stärker kandidatenorientiert ein Wahlsystem ist, desto stärker ist es anfällig für politische Korruption. ${ }^{12}$ Sie stehen u.a. damit im Widerspruch zu denjenigen Einschätzungen, die Listenwahlen als weniger effiziente Instrumente demokratischer Kontrolle ansehen, weil bei (geschlossenen) Listen der Wähler selbst keine Kontrolle über die konkrete Kandidatenauswahl besitzt.

12 Hiermit z.T. übereinstimmende Befunde finden sich bei Panizza $(2001,2003)$. 
Tabelle 4: Politische Korruption und Wahlregeln, WLS für 45 Demokratien

\begin{tabular}{|c|c|c|c|c|c|}
\hline \multirow{2}{*}{$\begin{array}{l}\text { Abhängige Variable: CPI } 2001 \\
\text { Ehemalige britische Kolonie }\end{array}$} & \multicolumn{5}{|c|}{ Std. Beta-Koeffizienten (t-Statistik) } \\
\hline & $\begin{array}{c}0,146 \\
(1,95)^{* *}\end{array}$ & $\begin{array}{c}0,186 \\
(2,32)^{* *}\end{array}$ & $\begin{array}{c}0,182 \\
(2,44)^{* *}\end{array}$ & $\begin{array}{c}0,180 \\
(2,41)^{* *}\end{array}$ & $\begin{array}{c}0,198 \\
(2,61)^{* * *}\end{array}$ \\
\hline GDP per Capita & $\begin{array}{c}0,822 \\
(10,62)^{* * *}\end{array}$ & $\begin{array}{c}0,865 \\
(10,39)^{* * *}\end{array}$ & $\begin{array}{c}0,852 \\
(11,15)^{* * *}\end{array}$ & $\begin{array}{c}0,867 \\
(11,05)^{* * *}\end{array}$ & $\begin{array}{c}0,877 \\
(11,18)^{* * *}\end{array}$ \\
\hline $\begin{array}{l}\text { Protestantischer } \\
\text { Bevölkerungsanteil }\end{array}$ & $\begin{array}{c}0,217 \\
(2,91)^{* * *}\end{array}$ & $\begin{array}{c}0,215 \\
(2,91)^{* * *}\end{array}$ & $\begin{array}{c}0,221 \\
(3,06)^{* * *}\end{array}$ & $\begin{array}{l}0,225 \\
(3,1)^{* * *}\end{array}$ & $\begin{array}{c}0,222 \\
(3,09)^{* * *}\end{array}$ \\
\hline Ballot & & $\begin{array}{l}-0,107 \\
(-1,33)\end{array}$ & & & \\
\hline Pool & & & $\begin{array}{c}-0,145 \\
(-1,99)^{*}\end{array}$ & & \\
\hline Vote & & & & $\begin{array}{c}-0,146 \\
(-1,96)^{*}\end{array}$ & \\
\hline (Ballot + Pool + Vote $)$ & & & & & $\begin{array}{l}-0,164 \\
(-2,173)^{* *}\end{array}$ \\
\hline \# Nobs & 47 & 47 & 47 & 47 & 47 \\
\hline Adj. $R^{2}$ & 0,76 & 0,76 & 0,77 & 0,77 & 0,78 \\
\hline
\end{tabular}

Quellen: Transparency International; Wallack et al. 2002; Persson et al. 2001; ${ }^{* * *} \mathrm{p}<0,01 ;{ }^{* *} \mathrm{p}<0,05 ;{ }^{*} \mathrm{p}<$ 0,1 .

\section{Schluss}

Korruptionsskandale erscheinen in der Öffentlichkeit oft als Parteienskandale. Sie fördern regelmäßig "Politikverdrossenheit" in der Bevölkerung. Doch nach dem hier dargelegten Argument basiert dies im Wesentlichen auf einer falschen Zurechnung. Parteien haben im Regelfall ein hohes Interesse daran, Kontrolle über ihre Kandidaten zu gewinnen, um eine „Parteireputation“ für Vertrauenswürdigkeit zu erwerben (Carey/ Shugart 1995; Kreps 1996). Parteien sind daher oft eher aktive Akteure in der Bekämpfung als Profiteure der politischen Korruption. Nicht dort, wo Parteien stark sind, sondern dort, wo sie nur schwache Kontrolle über ihre Kandidaten ausüben können, blüht die politische Korruption. Das klingt im Kontext der aktuellen öffentlichen Diskussionen ketzerisch, ist aber keineswegs eine neue These. Schon vor mehr als hundert Jahren konnte man die Einsicht gewonnen haben: „partisanship and corruption are fundamentally antagonistic principles. Partisanship tends to establish a connection based upon an avowed public obligation, while corruption consults private and individual interests which secrete themselves from view and accountability of any kind. The weakness of party organization is the opportunity of corruption " (zitiert nach Gerring/ Thacker 2004: 321). Die hier präsentierten empirischen Befunde legen nahe, dass es für Wähler anscheinend einfacher ist, Parteien zu beobachten, als Kandidaten. Gegenüber der ökonomischen Sichtweise, die die Persönlichkeitswahl als demokratisches Äquivalent zur Konsumentensouveränität sieht, muss betont werden, dass es im politischen Markt an einem einfachen Informationsträger wie dem Preis üblicherweise fehlt. Findet der politische Wettbewerb zwischen Parteien statt, und nicht zwischen Kandida- 
ten, scheint nach der vorliegenden empirischen Evidenz tatsächlich ein inverses Verhältnis zwischen politischem Wettbewerb und politischer Korruption zu herrschen. Würde man populären Vorschlägen folgen, politische Korruption durch die Etablierung eines direkteres Verhältnisses zwischen Wählern und Abgeordneten, etwa durch die Abschaffung der Listenwahl, einzudämmen, bestehen daher gute Chancen, genau entgegen gesetzte Effekte hervorzurufen.

\section{Literatur}

Abed, Geoerge T./Gupta, Sanjeev (Hrsg.), 2002: Governance, Corruption \& Economic Performance. Washington, D.C.

Ades, Alberto/Di Tella, Rafael, 1999: Rents, Competition and Corruption, in: American Economic Review 89, 982-993.

Bardhan, Pranab, 1997: Corruption and Development: A Review of Issues, in: Journal of Economic Literature 35, 1320-1346.

Beck, Thorsten/Clarke, George/Groff, Alberto/Keefer, Philip/Walsh, Patrick, 2002: New Tools and New Tests in Comparative Political Economy: The Database of Political Institutions. The World Bank.

Besley, Timothy/MacLaren, John, 1993: Taxes and Bribery: The Role of Wage Incentives, in: Economic Journal 103, 119-141.

Bollen, Kenneth, 1993: Liberal Democracy: Validity and Method Factors in Cross-National Measures, in: American Journal of Political Science 37, 1207-1230.

Cain, Bruce/Ferejohn, John/Fiorina, Morris, 1987: The Personal Vote: Constituency Service and Electoral Independence, Cambridge, Mass.

Carey, John M./Shugart, Matthew S., Matthew S., 1995: Incentives to Cultivate a Personal Vote: A Rank Ordering of Electoral Formulas, in: Electoral Studies 14(4), 417-439.

Cox, Gary W., 1987: The Efficient Secret - the Cabinet and the Development of Political Parties in Victorian England. New York.

Fearon, John D., 1999: Electoral Accountability and the Control of Politicians: Selecting Good Types versus Sanctioning Poor Performance, in: Przeworski, Adam/Stokes, Susan C./Manin, Bernard (Hrsg.), Democracy, Accountability, and Representation. New York, 55-97.

Fisman, Raymond/Gatti, Roberta, 2002: Decentralization and Corruption: Evidence across Countries, in: Journal of Public Economics 83, 325-345.

Gerring, John/Thacker, Strom C., 2004: Political Institutions and Corruption: The Role of Unitarism and Parliamentarism, in: British Journal for Political Science 34, 295-330.

Golden, Miriam, 2003: Electoral Connections: The Effects of the Personal Vote on Political Patronage, Bureaucracy and Legislation in Postwar Italy, in: British Journal of Political Science $33,189-212$.

Golden, Miriam/Chang, Eric C., 2001: Competitive Corruption, Factional Conflict and Political Malfeasance in Postwar Italian Christian Democracy, in: World Politics 53, 588-622.

Hine, David, 1996: Political Corruption in Italy, in: Little, Walter (Hrsg.), Political Corruption in Europe and Latin America. London, 137-157.

Kaufmann, Daniel/Kray, Aart/Zoido-Lobatón, Pablo, 2002: Governance Matters II. Updated Indicators for 2000/01. Policy Research Working Paper 2772. The World Bank.

Kreps, David M., 1996: Corporate Culture and Economic Theory, in: Kreps, David M. (Hrsg.), Firms, Organizations and Contracts. New York, 221-275.

Kunicova, Jana, 2000: Are Presidential Systems more Corrupt? Manuskript, Yale University.

Kunicova, Jana/Rose-Ackerman, Susan, 2002: Electoral Rules as Constraints on Corruption: The Risks of Closed-list Proportional Representation. Manuskript, Yale University.

Lambsdorff, Johann Graf, 1999: Corruption in Empirical Research - A Review, <http://www. transparency.org/working_papers/lambsdorff/lambsdorff_eresearch.html>, 7.01.2003. 
Lambsdorff, Johann Graf, 2002: Background Paper to the 2002 Corruption Perception Index, <http://www.transparency.org/cpi/2002/dnld/cpi2002.methodology.pdf>, 7.1.2003.

La Porta, Rafael et al., 1997: Trust in Large Organizations, in: American Economic Association, Papers and Proceedings 87, 333-338.

La Porta, Rafael et al., 1998: The Quality of Government. NBER working paper 5661. National Bureau of Economic Research. Cambridge, Mass.

Lijphart, Arend, 1999: Patterns of Democracy. New Haven.

Manow, Philip, 2002: Was erklärt politische Patronage in den Parteiensystemen Westeuropas? Defizite politischen Wettbewerbs oder formative Phasen demokratischer Massenmobilisierung?, in: Politische Vierteljahresschrift 43, 20-45.

Manow, Philip, 2003: Politische Korruption als Gegenstand der Politikwissenschaft - Eine Kritik des Forschungstandes, in: von Arnim, Hans-Herbert (Hrsg.), Korruption - Netzwerke in Politik, Ämtern und Wirtschaft. München, 239-273.

Mauro, Paolo, 1995: Corruption and Growth, in: Quarterly Journal of Economics 110, 681-712.

Montinola, Gabriella R./Jackman, Robert W., 2002: Sources of Corruption: A Cross-Country Study, in: British Journal of Political Science 32, 147-170.

Nohlen, Dieter, 2000: Wahlrecht und Parteiensystem. Opladen.

Panizza, Ugo, 2001: Electoral Rules, Political Systems, and Institutional Quality, in: Economics and Politics 13(3), 311-342.

Panizza, Ugo, 2003: Electoral Rules and Corruption, in: Transparency International: Global Corruption Report 2003, 317-319.

Persson, Torsten/Tabellini, Guido/Trebbi, Francesco, 2001: Electoral Rules and Corruption. NBER Working Paper 8154. National Bureau of Economic Research. Cambridge, Mass.

Persson, Torsten/Tabellini, Guido/Trebbi, Francesco, 2003: Electoral Rules and Corruption, in: Journal of the European Economic Association 1(4), 958-989.

Rae, Douglas W., 1971: The Political Consequences of Electoral Laws. New Haven.

Sandholtz, Wayne/Koetzle, William, 2000: Accounting for Corruption: Economic Structure, Democracy, and Trade, in: International Studies Quarterly 44, 31-50.

Sandholtz, Wayne/Gray, Mark M., 2003: International Integration and National Corruption, in: International Organization 57, 761-800.

Schiavo-Campo, Saluatorelde Tommaso, Giulio/Mukherjee, Amitabha, 1997: An International Statistical Survey of Government employment and Wages. World Bank Working Paper 1806, August.

Shugart, Matthew. S., 2001: Electoral "Efficiency" and the Move to Mixed-member Systems, in: Electoral Studies 20, 173-193.

Strøm, Kaare, 1989: Inter-Party Competition in Advanced Democracies, in: Journal of Theoretical Politics 3, 277-300.

Strom, Kaare, 1990: A Behavioral Theory of Competitive Political Parties, in: American Journal of Political Science 2, 565-598.

Taagepera, Rein/Shugart, Matthew S., 1989: Seats \& Votes. The Effects and Determinants of Electoral Systems. New Haven.

Treisman, Daniel, 2000: The Causes of Corruption: A Cross-national Study, in: Journal of Public Economics, 399-457.

Treisman, Daniel, 2002: Decentralization and the Quality of Government. Manuskript, UCLA.

Van Rijckeghem, Caroline/Weder, Beatrice, 1997: Corruption and the Rate of Temptation: Do Low Wages in the Civil Service Cause Corruption? IMF Working Paper, June.

Wallack, Jessica Seddon/Gaviria, Alejandro/Panizza, Ugo/Stein, Ernesto, 2002: Political Particularism around the World. Inter-American Development Bank, Working Paper 463. 